\title{
A Unique Syndrome of Gemination and External/ Internal Root Resorptions in One Dental Arch: A Case Report
}

ISSN: 2637-7764

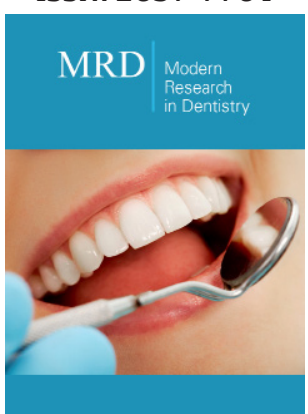

*Corresponding author: Kazemi Reza B, Associate Professor, UCONN Health School of Dental Medicine, USA

Submission: 海 August 08, 2020

Published: 佔 May 03, 2021

Volume 6 - Issue 3

How to cite this article: Pray Nickolas, Raghavendra Sree, Kazemi Reza B. A Unique Syndrome of Gemination and External/Internal Root Resorptions in One Dental Arch: A Case Report. Mod Res Dent. 6(3). MRD. 000636. 2021.

DOI: 10.31031/MRD.2021.06.000636

Copyright@ Kazemi Reza B, This article is distributed under the terms of the Creative Commons Attribution 4.0 International License, which permits unrestricted use and redistribution provided that the original author and source are credited.

\author{
Pray Nickolas ${ }^{1}$, Raghavendra Sree ${ }^{2}$ and Kazemi Reza B ${ }^{3 *}$ \\ ${ }^{1}$ Pediatric Dentistry Resident, UCONN Health School of Dental Medicine, USA \\ ${ }^{2}$ Assistant Clinical Professor, UCONN Health School of Dental Medicine, USA \\ ${ }^{3}$ Associate Professor, UCONN Health School of Dental Medicine, USA
}

\begin{abstract}
Dental literature, considering a unique syndrome of two distinct developmental disruptions; fusion and gemination teeth and morphological irregularities; external/internal root resorptions caused by trauma or disease in one dental arch was reviewed.

The PubMed and Google Scholar citations revealed only one report of fusion and internal resorption in the same arch/tooth. However, the present case report's dental inquiry revealed a history of a geminated maxillary left lateral incisor tooth [\# 10] in addition to a dental trauma more than 20 years ago, where both teeth [\#s $08 \& 09$ ] were traumatized. Tooth [\# 09] was avulsed and replanted back into dental alveoli under an unusual circumstance. The clinical and radiograph analysis of these two teeth revealed their enduring involvement with the external/internal root resorptions as well. Patient reported, no other significant past dental history, except routine dental visits and treatments. The clinical and radiographical evaluation, findings, and diagnosis are discussed. The proposed treatment plans for management of the unilateral geminated tooth, external/internal root resorptions and clinical problem of severe occlusal wear provided as of Fall 2020 are also described.
\end{abstract}

\section{Introduction}

Developmental dental disorders and malformed teeth may be due to congenital, inherited, acquired or idiopathic reasons causing anomalies in tooth number, size, shape and structure. They are of special clinical awareness regarding to the complications of oral hygiene maintenance, dental treatment care and appearance as well [1-9].

Various terms have been used to describe dental developmental abnormalities resulting differentunusual morphologies as well, such as double tooth; fusion/synodontia, gemination/ twinning/schizodontia, and concrescence, dilaceration, etc. Relevant to the presented rare syndrome case, two terms are the main considerations. The fusion of teeth, which is characterized by the union of two adjacent teeth resulting in a reduction in the numberof teeth in the affected arch. Gemination is, when there is a division of a single tooth germ by invagination with the resultant incomplete or complete formation of two teeth [1-3,6-9].

The prevalence of these anomalies; fusion and gemination and two distinct morphological characteristics/patterns; inflammatory internal and external root resorptions and their physiologic/transient; have been individually distinguished and well documented in the dental literature [1,2,9-22]. However, the search via PubMed and Google Scholar revealed that such case only one time was reported back in over 42 years ago, which specifies as the combination of these two anomaly and root resorption in the same dental arch/tooth [1,1214]. The PubMedcitation search also indicated that the terms "fusion/fused and gemination/ geminated Teeth" have been extensively documented in the literature but distinguishing between fusion and gemination can somehow be challenging and often confusing [9,12-14]. However, as a significantlandmark for distinction of these two types of tooth anomalies, the major differential diagnosis is usually made by counting the number of teeth in the involved arch [6-9,10-14]. 
Fusion has been independently adjoining primary or permanent tooth germs, resulting in a reduction in the number of teeth in the affected arch [6-9,10-14]. The tooth gemination structuremay usually be one with two completely or incompletely separated crowns that mostly have a single root and root canal, $[7,8]$. In contrary, a geminated maxillary lateral incisor with two root canals has also been reported, (03). Double tooth and twinning are also used to describe this anomaly. Twinning is considered as the complete form of gemination anomaly that concludes in the formation of two separate teeth from a single tooth bud resulting one extra tooth in the arch $5,9,14]$. As a result, if the number of teeth is not altered or may be increased by one extra toothin the same arch, this case(s) would be considered as a gemination $[3,5,8,9,11,13,14]$.

Developmental dental anomalies like gemination may cause functional, orthodontic, endodontic and esthetic impairments and represent a challenge for the patients and dentists as well. In most cases, a multidisciplinary approach is required to obtain the more appropriate and applicable treatment option(s) for a successful outcome $[11,15,19,20-22]$.

Considering all of the aforementioned, the clinicians must be depending on both clinical examination and radiograph analysis to make the final judgment and best diagnosis between these two abnormalities.

Nevertheless, the etiology of these developmentally disturbed teeth also remains somehow unclear although genetic and environmental bases have been suggested $[3,9,11,13,14]$. As a conclusion, considering many different descriptions for these two anomalies, the following twodevelopmental disorders involved in the presented syndrome case are:

\section{Tooth fusion}

Tooth fusion as a form of a developmental anomaly that arises through fusion of dentin and enamel of two or more developing, but normally separated teeth, at the crown level, causing theformation of a tooth with an enlarged clinical crown. This process may be either complete or incomplete. The most common landmark to distinct between these two anomalies is related to counting the number teeth in the same arch. The fusion of teeth is characterized by a union of two adjacent teeth. The differential diagnosis is usually made by counting the number of teeth resulting in a reduction in the number in the affected arch [1] On some occasions, two independent pulp chambers and root canals have also been reported [3].

\section{Tooth gemination}

Tooth gemination that is also called twinning or double tooth [7$9,13]$, comprised of two teeth that is considered as morphologically dental anomaly. It is, in fact, the partial developmentof two teeth from a single tooth bud/germ following incomplete division. The geminated tooth is normally oversized or abnormally shaped tooth. Gemination occurs when a single bud splits and forms two teeth. Germination typically looks like a single enlarged tooth, so there is no difference in the tooth count $[6-8,10-13,19]$. Therefore, the differential diagnosis is usually made by counting the number of teeth in the arch, which is not altered in the cases of gemination or may rarely increase.

\section{External \& internal root resorptions}

Deciduous root resorption is a normal physiologic response to the eruption of permeant teeth, but the permanent dentition root resorption is normally a pathologic process. This phenomenonmay be respectfully classified into two distinct external and internal root resorptions. The external resorption initiates from the Periodontal Ligament (PDL), where the inflammation of internal resorption originates from the dental pulp tissue. The inflammation generated by the cariogenic oral microorganisms and trauma are two distinct factors causing the root resorptions $[1,16-19,21,22]$.

\section{Case Presentation}

For this case presentation, a consent was obtained for the use of patient's photographs and other visual documentations. A 68-yearold male presented to the University of Connecticut School of Dental Medicine's clinics for comprehensive dental care but requested prioritizing repair of worn-down anterior teeth. Patient reports that anterior teeth [\#s 07, 08, 09] have become severely chipped over the past $3+$ years. Edges of chipped teeth are sharp and cause discomfort during eating as well as speaking.

Patient's medical history was reviewed. Patient reported that he quit a 15-year smoking habit over 5 years ago. Patient is clinically obese and in 2017 was diagnosed with obstructive sleep apnea; and ever since uses a CPAP machine at night. Patient reported type 2 diabetes that is wellcontrolled with his most recent $\mathrm{HbA1C}$ at 6.2. Patient also reported a 30-year history of Gastroesophageal Reflux Disease (GERD). Patient was hospitalized back in January 2017 for a heartattack where he subsequently received coronary artery bypass surgery. Patient has followed a routine of prescription anti-anxiety medications given the noted stressful nature of his job for the past 10 years. Patient's health is currently well controlled for all ailments by taking a series of medications; Lexapro, Aspirin, Atorvastatin, Gabapentin, Metformin, Metoprolol Succinate, Omeprazole, and Ranitidine HCl. Patient has denied any unusual diet, allergic history.

Patient's dental history was also reviewed. Patient reports past dental trauma about 20 years ago, where tooth [\# 09] was avulsed and then replanted back into socket immediately after trauma by the patient. During the fall, tooth [\# 08] was also traumatized. Patient denies dental consultation after trauma occurred nor did he have professional help re-implanting tooth [\# 09].Patient reports, except routine dental care and treatments, no other significant past dental history and no family history of significant dental problems.

Radiograph and intraoral examinations reveal worn-down teeth [\#s 07, 08 and 09] (Figures 1-5) as well as tooth gemination [\# 10] (Figures 1-7). The evaluation of periapical radiographs taken of teeth [\#s 07, 08, 09, and 10] presents with no evidence of periapical radiolucency. Tooth [\# 08], however, shows significant internal root resorption to the mid-way and calcified the apical half of the root to the apex. Tooth [\# 09] also shows a significant external/internal root resorption which is more evident on the distal aspect of the tooth, (Figure 7). Both merged segments of geminated tooth [\#10] (Figure 6), shows almost completely calcified root canals to the apex. This calcification is also evident in tooth [\# 07] (Figure 7). 


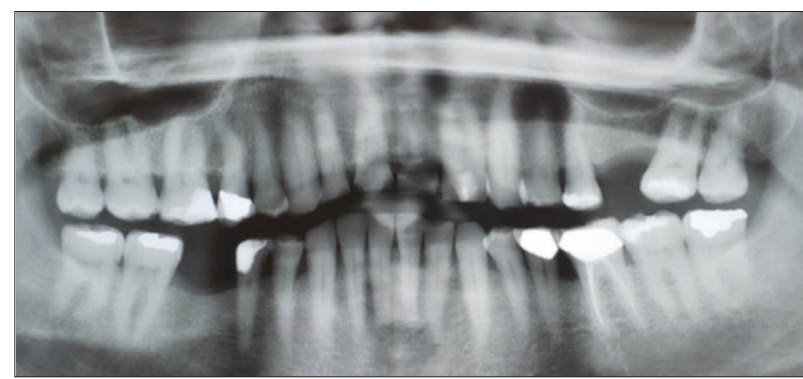

Figure 1: Panoramic radiograph reveals no reduction in maxillary arch dentition (Counted all 16 teeth including the extracted \# 14 and geminated tooth \# 10, as a single tooth).

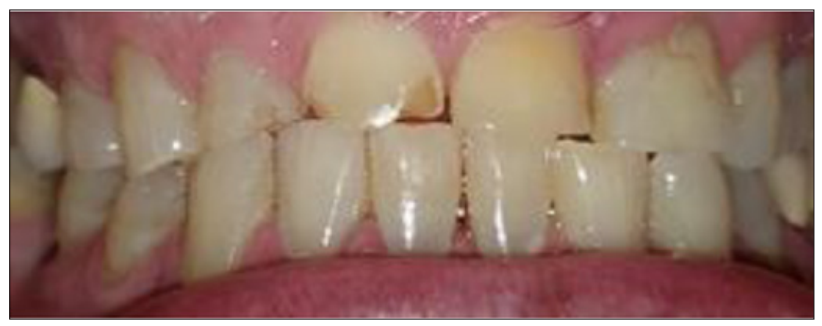

Figure 2: Facial / Labial view oversized crown of geminated tooth \# 10 end to end incisal contacts with chipped incisal edges of anterior teeth.

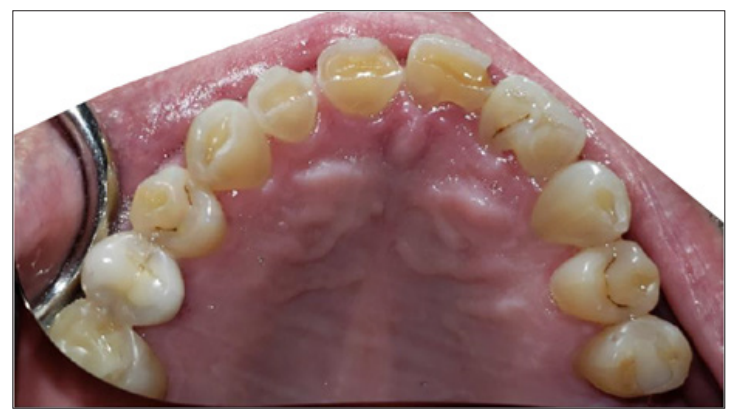

Figure 3: Maxillary occlusal view heavy attrition on incisal edges of anterior teeth oversized/abnormally shaped of geminated tooth \# 10.

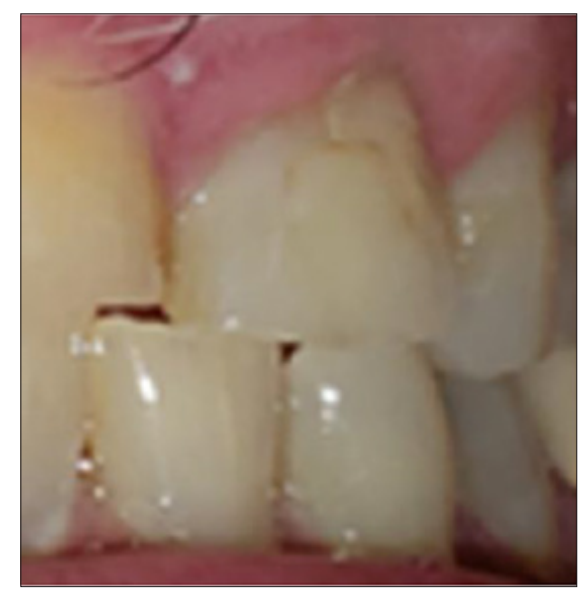

Figure 4: Facial / Labial view oversized/abnormally shaped geminated tooth \# 10 .

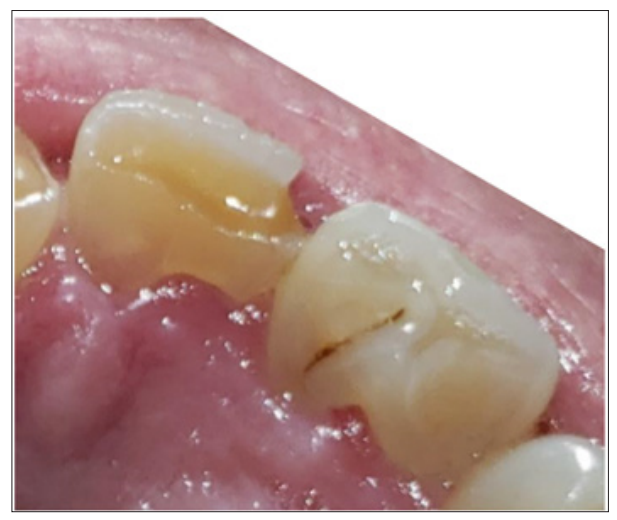

Figure 5: Linguo-incisal view geminated / double tooth \# 10 heavy attrition on incisal edge / fracture on Disto-Facio-Linguo-Incisal (DFLI) of tooth \# 09.

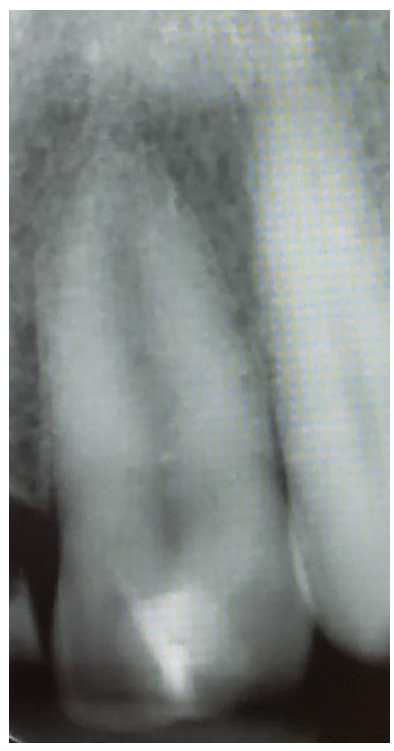

Figure 6: Germinated/Double tooth with completely calcified root canals.

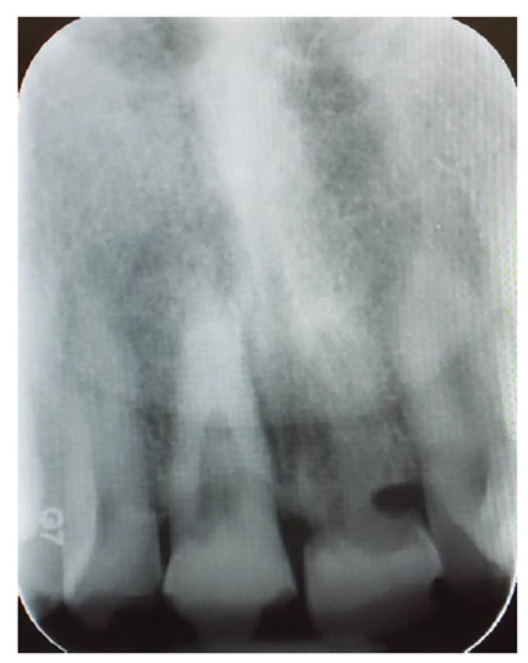

Figure 7: Teeth \#s $08 \& 09$ radiographic evidence of external / internal root resorptions \& partially calcified root canal. 
An endodontic consultation was obtained to evaluate the treatment options for teeth [\#s 08 and09]. In addition to the calcified apical half of the root canal of tooth [\# 08], a communication between the external and internal root resorption appears more towards the cervical third zoneof the root. Tooth [\# 09] has external root resorption with an extended resorbed canal. It was stated that root canal treatment is not indicated for either tooth and they should be monitored and planned for the extraction.

The patient neither wishes to extract and replace [\# 08 and \# 09] nor is interested in a conventional fixed or removable denture at this time. Pulp vitality tests were completed on [\#s 07, 08, 09 and 10]. EPT test showed positive responses for all four teeth: [\# 07(7), \# 08(42), \# 09(53), and \# 10(42)]. Cold test was negative for all teeth tested.

It was concluded that due to root resorption, teeth [\#s 08 and 09] are not the appropriate candidates for endodontic treatment. This was also included tooth [\# 07] that was not the appropriate candidate for the resin composite restorations due to the notable occlusal wear of the maxillary teeth and end-to-end occlusion. The patient exhibits heavy bruxism, and it was suggested that a mouth guard should be prescribed for nighttime use. A removable night guard was fabricated for the patient which will be worn in the evenings to prevent further damage to the anterior teeth. The night guard can also be customized to prevent snoring at night as well.

\section{Discussion}

The main reason of presenting this special case is to report a rare unique syndrome of two distinct developmental and morphological irregularities that have occurred in one dental arch. One is a unilateral gemination of maxillary left lateral incisor, as a result of developmentally disruption ofthe tooth, and the others are the evidence of external/internal resorptions involving both maxillary central incisors that could have been the result of trauma; causing the avulsion of tooth [\# 09] that was replanted and traumatizing tooth [\# 08] [23,24].

The literature review did not support adequate number of cases of this kind for a logical literature analysis. The clinical and radiographic evidence of the presented case confirmed its complexity that was challenging to differentiate between fusion and gemination. However, this article highlighted the important evidence for diagnosing between these two developmental anomaliesof teeth. It was a strong landmark of no tooth numbering count reduction of the maxillary dentalarch in favor of gemination.

The other important rationale for sharing this case is related to the fact that if the patient had reported the case to a health care provider within an appropriate time frame and/or the case was followed up later, the teeth could high likely be treated on time and saved.

Lastly, to confirm the above statement, by considering possible correlation between the trauma and the root canal calcification and/or external and internal root resorption, similar to this case, it is concluded that the maxillary anterior teeth, including the geminated tooth, have been impacted with these distinct disruptions. The importance of clarifying an early diagnosis of root resorption and/or calcification in relation to the different factors (patient's age, time of trauma and receiving possible emergency service, nature of trauma, etc.) would improve the plans for abetter prognosis by monitoring the traumatized teeth and rendering the required treatment(s) on an appropriate time as needed, $[23,24]$.

\section{Conclusion}

Upon the radiograph and clinical examinations of a patient referred to the UConn Heath Dental Clinic, the existence of a geminated tooth [\# 10], with the combination of external/internal rootresorptions on teeth [\#s 08 \& 09] following a trauma more than 20 years ago was confirmed.

Dental literature, considering such uncommon syndrome of distinct developmental disruption and root resorption caused by trauma or disease in a one dental arch was reviewed. The results revealed that a similar syndrome was only reported once over 42 years ago, which specified in the same dental arch/tooth.

The management of this uncommon syndrome case should be based on early observations, considering their clinical and radiographic evaluations. The present case had not followed such a protocol. Under current circumstances, their management is, therefore, mostly focused on a comprehensive dental treatment protocol based on the existing conditions, and future plans are depending on all limitations, in particular, during the COVID-19 pandemic and post pandemic eras [25]. Addition to the main concern of this manuscript, other complications including the bruxism, loss of VDO, exaggerated Curve of Spee and loss of significant tooth structure; the treatment planning will need to take a multidisciplinary approach. The patient does not want to address comprehensive treatment currently and wants the fabrication of an occlusal guard only.Considering the patient's lack of interest and/or socioeconomical limitations, this syndrome case should be monitored and revisited at a timely manner. Meanwhile, if any emergency arises, the case would be managed accordingly. Knowledge and attention to the complex morphology of thetooth will help the dental health care provider/dentist to make a more proper decision regardingto the treatment planning and rendering necessary treatments on a more appropriate manner.

\section{References}

1. Mehlman ES (1978) Management of a totally fused central and lateral incisor with internal resorption perforating the lateral aspect of the root. J Endod 4(6): 189-191.

2. Duncan WK, Helpin ML (1987) Bilateral fusion and gemination: a literature analysis and case report. Oral Surg Oral Med Oral Pathol 64(1): 82-87.

3. Romano N, Souza Flamini LE, Mendonça IL, Silva RG, Cruz Filho AM (2016) Geminated maxillary lateral incisor with two root canals. Case Rep Dent 2016: 3759021.

4. Shankar AA, Koshy AV, Duggal DS, Dandekar RC (2011) Synodontia with talon cusp: A case report and review of literature. Oral \& Maxillofacial Pathology Journal 2(2): 165-169.

5. Knezevic A, Travan S, Tarle Z, Sutalo JB, Jankovic B, et al. (2002) Double tooth. Coll Antropol 26(2): 667-672. 
6. Suroopa D, Warhadpande MM, Saurabh AR, Husain S, Tushar S (2015) Management of Syndontia between dilacerated permanent maxillary central incisor and supernumerary tooth with aid of cone-beam computed tomography. J Conserv Dent 18(2): 163-167.

7. Grover PS, Lorton L (1985) Gemination and twinning in permanent dentition. Oral Surg Oral Med Oral Pathol 59(3): 313-318.

8. Sekerci AE, Sisman Y, Ekizer A, Sahman H, Gusmus H, Aydinbelge M (2011) Prevalence of double (Fused/ Geminated) permanent teeth in Coppadocia region in Turkey-a study. Pak Oral \& Dent J 31: 17-22.

9. Nandini DB, Deepak BS, Selvamani M, Puneeth HK (2014) Diagnostic dilemma of a double tooth: A rare case report and review. J Clin Diagn Res 8(1): 271-272.

10. Ansari G, Golpayegani MV, Welbury R (2019) Chapter 4: Etiology and pathology of teeth disturbances: 21-61. Atlas of Pediatric Oral and Dental Developmental Anomalies, ( $1^{\text {st }}$ edn), John Wiley \& Sons Ltd, New York, USA.

11. Finkelstein T, Shapira Y, Bechor N, Shpack N (2015) Fused and geminated permanent maxillary central incisors: prevalence, treatment options, and outcome in orthodontic patients. J Dent Child (Chic) 82(3): 147-152

12. Vorwaller RA (2020) Developmental dental anomalies: prevalence and association with medical background. Thesis submitted for the degree of Master of Science in oral sciences in the Graduate College of the University of Illinois at Chicago, USA.

13. Lagarde M, Bonnet AL, Douangmala N, Traing M, Dursun E (2020) Simultaneous occurrence of triple teeth and double teeth in primary dentition: A rare case report and review of the literature. Clin Case Rep 8(7): 1277-1286.

14. Martinez F, Segura F, Paisano J (2018) Dental union abnormalities: literature review. Acta Scientific Dental Sciences 2(11): 36-40.
15. Faria MIA, Borges ÁH, Carneiro SM, Filho JMS, Segundo AS, et al. (2011) Endodontic treatment of dental formation anomalies. Rev Odonto Cienc 26(1): 88-91.

16. Tronstad L (1988) Root resorption-etiology, terminology, and clinical manifestations. Endod Dent Traumatol 4(6): 241-252.

17. Gividen SL (2016) Endodontics reference guide: Distinguishing differences between internal and external resorption.

18. Simons S (2014) Internal resorption: A brief review and case report.

19. Gartner AH, Mack T, Somerlott RG, Walsh LC (1976) Differential diagnosis of internal and external root resorption. J Endod 2(11): 329-334.

20. Consolaro A, Furquim LZ (2014) Tooth movement: A protocol for clinical management. Dental Press J Orthod 19(5): 19-26.

21. Bastos JV, de Souza Côrtes Mi, Goulart EMA, Colosimo EA, Gomez RS, et al. (2014) Age and timing of pulp extirpation as major factors associated with inflammatory root resorption in replanted permanent teeth. J Endod 40(3): 366-371.

22. Senışık NE, Koçer G, Kaya BÜ (2014) Ankylosed maxillary incisor with severe root resorption treated with a single-tooth dento-osseous osteotomy, vertical alveolar distraction osteogenesis, and mini-implant anchorage. Am J Orthod Dentofacial Orthop 146(3): 371-384.

23. Dhinesh K, Delphine PA (2018) Calcified canal and negotiation-a review. Research J Pharm and Tech 11(8): 3727-3730.

24. Soares AJ, Souza GA, Pereira AC, Vargas Neto J, Zaia AA, et al. (2015) Frequency of root resorption following trauma to permanent teeth. J Oral Sci 57(2): 73-78.

25. Dal Molin K, Loo C, Kazemi RB, Weinstein G (2021) The practice of dentistry amidst the COVID-19 pandemic. Clin Dent Rev 5(4): 1-15. 